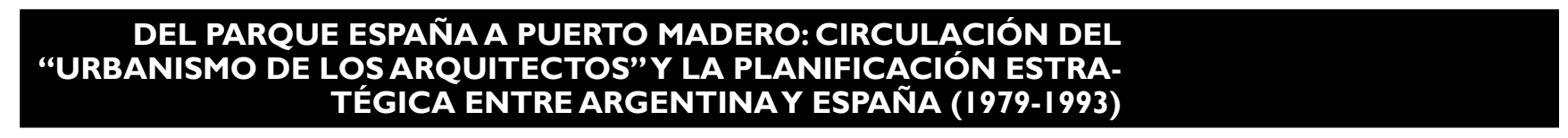

\title{
Guillermo Jajamovich
}

Doctor en Ciencias Sociales (UBA), becario posdoctoral CONICET con sede en el Instituto de Estudios de América Latina y el Caribe, Facultad de Ciencias Sociales, Universidad de Buenos Aires. Docente de grado y posgrado (UNLP - UTDT). guillermopazjajamovich@, gmail.com

ISNN 1666-6I86. Volumen 12 N 12 (junio 20I2) pp. 007-025 - Recibido: 14-08-11 Aprobado: 08-03-12 


\title{
Resumen
}

El artículo analiza algunas características de la circulación internacional de ideas urbanas a partir del despliegue de una serie de iniciativas urbanísticas en Buenos Aires y Rosario entre 1979 y 1993 . Solo algunas de las numerosas ideas y estrategias del debate internacional "llegan" a Buenos Aires y Rosario, y lo hacen de una manera desarticulada. Así, se abordan algunos elementos del discurso "español" en torno al "urbanismo de los arquitectos" y la planificación estratégica. Se analiza su contexto de recepción, revisando el debate disciplinar y considerando las condiciones políticas y económicas en las aquellas ideas "llegan" y los actores públicos y privados que participan en estos procesos. Respecto de Rosario, se analiza la presencia del arquitecto catalán Oriol Bohigas y su anteproyecto para Parque España realizado en el año 1979. En Buenos Aires, se examina el inicio de la operación Puerto Madero.

\section{Palabras clave}

Circulación de ideas, urbanismo de los arquitectos, planificación estratégica, Buenos Aires, Rosario.

\begin{abstract}
$\underline{\text { Abstract }}$
The aim of this paper is to analyse various characteristics of the international circulation of urbanistic ideas from the point of view of their applications in Buenos Aires and Rosario between 1979 and 1999. Only some of the numerous ideas and strategies of the international debate arrived in Buenos and Rosario, and they did so in an unarticulated manner. I take into account some elements of the Spanish discourse around urban projects and urban management. The context of the reception of these ideas requires a review of the disciplinary debate around urban projects and urban management as well as the political and economic conditions in which they arrived and the public and private actors involved. For Rosario, I look into the presence of the Catalan architect Oriol Bohigas and his project for Parque España (1979). In Buenos Aires I examine the revitalized industrial area of Puerto Madero.
\end{abstract}

Key words

Circulation of ideas, architect's urbanism, strategic planning, Buenos Aires, Rosario. 
Del parque España a Puerto Madero: circulación del "urbanismo de los arquitectos" y la planificación estratégica entre Argentina y España (1979-1993)

\section{INTRODUCCIÓN}

El presente artículo propone analizar algunas características de la circulación internacional de ideas y políticas urbanas a partir de una serie de iniciativas desplegadas en las ciudades de Buenos Aires y Rosario entre 1979 y 1993. Investigadores como PierRE BourdieU señalan ciertas características de los procesos de circulación internacional de ideas. Según el sociólogo francés, los intercambios internacionales están sometidos a un cierto número de factores estructurales que son generadores de malentendidos: "... el hecho de que los textos circulen sin su contexto (...) que no importen con ellos el campo de producción (...) del cual son el producto, y de que los receptores, estando ellos mismos insertos en un campo de producción diferente, los reinterpreten en función de la estructura del campo de recepción, es generador de formidables malentendidos...” (BOURDIEU, 2000: 161).

Solo algunas de las numerosas ideas, políticas y estrategias del debate internacional "llegan" a Buenos Aires y Rosario, y lo hacen de una manera desarticulada. En esta ocasión, abordaré algunos elementos del discurso español en torno al "urbanismo de los arquitectos" y la planificación estratégica. ${ }^{1}$ Analizar el contexto de recepción de aquellas ideas requiere la revisión del debate disciplinar en torno a los proyectos urbanos y la gestión urbana, el análisis de las condiciones políticas y económicas en las cuales aquellas ideas y perspectivas "llegan", así como la consideración de los actores públicos y privados que participan en estos procesos.

En relación con la ciudad de Rosario, analizaré la presencia del arquitecto catalán ORIOL Bohigas y su anteproyecto para Parque España realizado junto a sus socios MartorelL y MAKCAY.

Desarrollado en un contexto de dictadura militar, en el año 1979, aquel anteproyecto introduce desde una perspectiva arquitectónica una serie de críticas a la planificación urbana. Como veremos, propone una intervención limitada y puntual a partir de herramientas provenientes del ámbito de la arquitectura. A su vez, cabe señalar que este anteproyecto es previo al proceso a través del cual Barcelona se consagra como experiencia exitosa y una serie de actores participantes se encargan de su difusión internacional.

En torno a Buenos Aires, examinaré los comienzos de la operación Puerto Madero, en la que ingresa con mayor fuerza una serie de elementos ligados a la urbanística de los promotores y la planificación estratégica corriendo el eje, aunque retomando elementos de la discusión disciplinar. Uno de los puntos clave en esa dirección es el desarrollo en
1- Si bien no será esta la oportunidad de ampliar la cuestión, los debates y experiencias del contexto español deben entenderse como parte de discusiones más amplias que trascienden aquel territorio. Entre otras direcciones recorridas por el "urbanismo de los arquitectos", pueden mencionarse los extendidos contactos culturales entre arquitectos catalanes e italianos (Sainz Gutiérrez, 2006). 
2- Si bien el anteproyecto es del año 1979, la inauguración de solo un sector se producirá en 1993, luego de una extensa serie de problemas vinculados con el financiamiento y la propiedad de la tierra donde se asentaría.
1989 de la figura de la Corporación Antiguo Puerto Madero como elemento para destrabar la superposición jurisdiccional en el área, explicitando sus vínculos con una modalidad público-privada de gestión. Otro elemento novedoso y que se vincula nuevamente con el escenario internacional tiene que ver con el encargo en 1990 de un masterplan para Puerto Madero a un equipo catalán.

Finalmente, pese a las diferencias de escala entre Parque España (cuyo anteproyecto inicial se extendía en doce hectáreas) y Puerto Madero (cuyo proyecto final, aún no concluido, contempla 170 hectáreas), el presente trabajo confronta algunos aspectos de ambas operaciones, prestando especial atención a los actores públicos y privados que participan, así como al desplazamiento en los referentes y asesores extranjeros y sus perspectivas de intervención, que van desde ideas ligadas a intervenir en la ciudad a partir de proyectos de arquitectura hasta posiciones posteriores que trazan puentes entre el ámbito de la ciudad y el de las empresas. Así, a partir de diferentes entramados de actores, ideas similares del debate internacional se transculturizan de modo diverso, con efectos distintos en Buenos Aires y Rosario.

\section{ROSARIO Y EL PARQUE ESPAÑA: “URBANISMO DE LOS ARQUITECTOS” Y CRÍTICAS A LA PLANIFICACIÓN URBANA}

En el año 1979, el arquitecto catalán Oriol Bohigas y sus socios MARTORELl y MAcKaY (MBM) desarrollan el anteproyecto para el Parque España en Rosario. ${ }^{2}$ La iniciativa del Parque España surge a partir de la Federación de entidades españolas de la provincia de Santa Fe, la Municipalidad de Rosario y el Consulado general de España en Rosario, colaborando a su vez el Centro de Arquitectos de Rosario. Seleccionado por la Municipalidad de Rosario, el sector donde se desarrollará el anteproyecto estaba próximo al área central, encontrándose desactivado de sus usos ferroportuarios. En el anteproyecto, los nuevos usos serán principalmente recreativos y se vincularan asimismo con instituciones culturales y educativas.

El anteproyecto de MBM despliega, desde una perspectiva estrictamente arquitectónica, una serie de temas del debate internacional, tales como la crítica a la planificación urbana. Así, no desarrollan una propuesta global y abstracta para la totalidad de la costa de Rosario. Por el contrario, proponen un anteproyecto limitado para una parte específica de la costa, asumiendo los límites de la intervención desde una propuesta ligada al diseño urbano, esperando que irradie futuras intervenciones. En esa dirección, el proyecto pretendía ser extendido en posteriores fases. Al mismo tiempo, el anteproyecto da cuenta de una nueva 
Del parque España a Puerto Madero: circulación del "urbanismo de los arquitectos" y la planificación estratégica entre Argentina y España (1979-1993)

mirada sobre lo natural en el debate internacional al recuperar el río, en este caso el Paraná, y las barrancas adyacentes como valor urbano y cultural a partir de una intervención arquitectónica atenta al contexto en que se desarrolla.

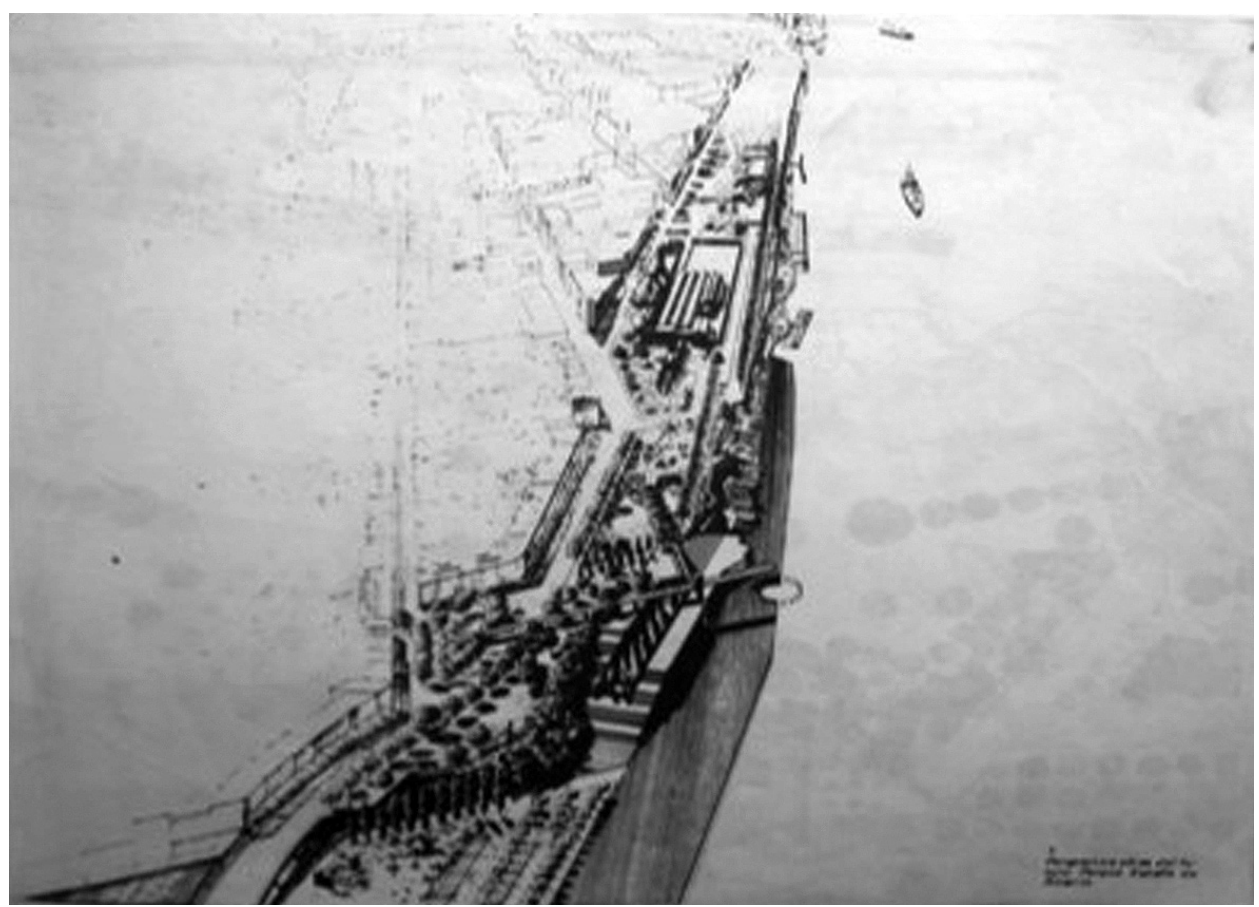

Junto al desarrollo del anteproyecto, en mayo del año 1979, BoHIGAS dictó en Rosario unas conferencias sobre temas de arquitectura alrededor de tres ejes: "La arquitectura en la ciudad", "La ciudad en la arquitectura" y "Persistencia tipológica" presentando en relación con ellos ejemplos de las obras de MBM. Allí desarrolló una serie de críticas a la "... solución urbana que el Movimiento moderno ha provocado..." (Elguezabal, 1979: 56), ligadas a una extensa discusión que se desarrollaba previamente en el contexto internacional, en el cual los debates en Italia alrededor del neorracionalismo y el rol de la arquitectura en la ciu- 


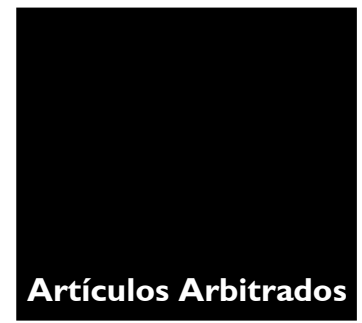

\section{Guillermo Jajamovich}

3- Si bien analizo en forma conjunta el contenido de las conferencias de Bohigas y las características del entonces futuro anteproyecto del Parque España, esto no significa que las ideas en arquitectura $y$ en urbanismo sean necesariamente equivalentes en producciones escritas y en proyectos arquitectónicos.

Fachadas y secciones del edificio. Fuente: revista A/mbiente, $N .^{\circ} 46$, julio1985, La Plata dad y su extensión en el continente europeo ocupaban un lugar relevante (SAINZ GuTIÉRREZ, 1999, 2006). Según BoHIGAS, cada proyecto debería ser una respuesta al entorno urbano o una propuesta de entorno urbano, señalando así la "... propuesta de reencuentro con las características urbanas tradicionales...” (ELGUEZABAL, 1979: 56). Esto resultará evidente en la terraza del Parque España adaptada, como todo el anteproyecto, a las condiciones topográficas y funcionando como un espacio público que permite el uso y disfrute del río, que previamente se encontraba, en aquella zona, desconectado de la ciudad, excepto en torno de la actividad económica alrededor del puerto. ${ }^{3}$ El propio edificio del parque España permitirá caminar por su interior, por las paredes laterales, que son escalinatas y por el techo, que es simultáneamente la pasarela peatonal del parque. A su vez, una serie de elementos tradicionales de la ciudad de Rosario eran reutilizados en el anteproyecto:

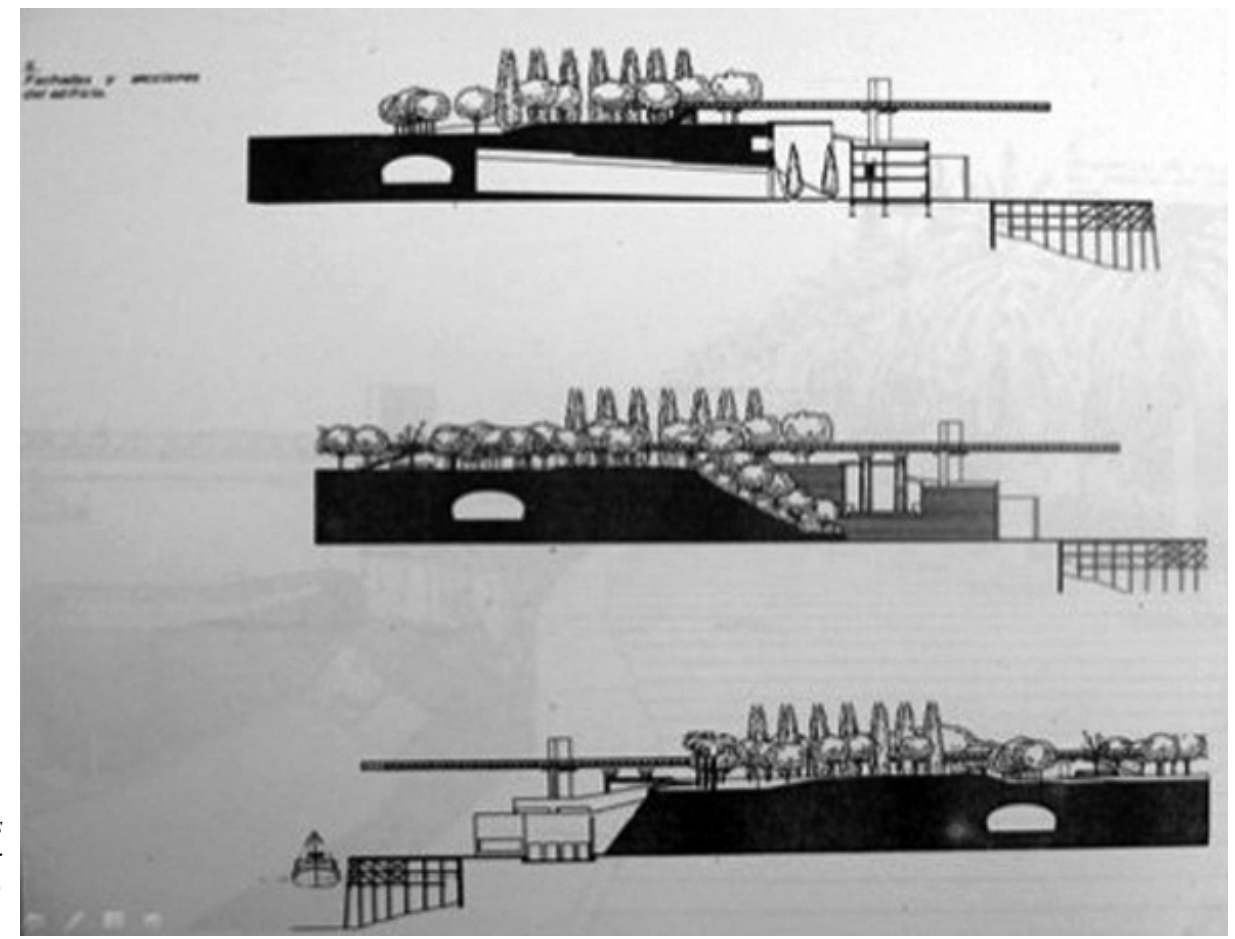


Del parque España a Puerto Madero: circulación del "urbanismo de los arquitectos" y la planificación estratégica entre Argentina y España (1979-1993)

MBM recuperaba unos túneles previos al desarrollo de la actividad portuaria, aspectos de la vegetación local y edificios ferroviarios restaurados, entre otros aspectos. ${ }^{4}$

Pero mientras en España estas perspectivas de intervención urbana que desde lo arquitectónico impugnaban la planificación urbana se ligaban a los nuevos ayuntamientos democráticos, aunque fueran producto de un debate que los precediera (SAINZ GUTIÉRREZ, 2006), el anteproyecto de MBM en Rosario introducía estas miradas en un contexto de restricciones democráticas en el que el espacio para discusiones urbanas, arquitectónicas, teóricas o de cualquier tipo era reducido. En aquel contexto, el proyecto del Parque España era presentado por la Municipalidad de Rosario como una respuesta al déficit cuantitativo de espacio verde en la ciudad. Pero posteriormente, con el retorno de la democracia y con unas renovadas Municipalidad y Secretaría de Planeamiento Urbano, un nuevo enfoque emergería vinculando proyectos como el del Parque España con la cuestión del espacio público y las posibilidades de realizar intervenciones urbanas a partir de proyectos de arquitectura. $^{5}$

En la materialización del Parque España pueden señalarse otras características de los procesos de circulación de ideas. La etapa de construcción del proyecto fue completada por dos estudios de arquitectura de Rosario. ${ }^{6} \mathrm{El}$ anteproyecto de MBM incluía un mirador que trascendía las piezas arquitectónicas atándolas por un lado a la cuadrícula de la ciudad y por otro, desembocando en el río con una cascada de agua, que simboliza “... el constante fluir espiritual entre ambas culturas..." (Estudio MBM, MARTORELl, BohigAS, MAC KAY; Estudio H; Quiroga, Horacio y asociados, arqs., 1983: 73). Como se sabe, la cuadrícula es un elemento estructural de diversas ciudades, muchas veces ligada a la presencia española en los tiempos de la Colonia. Dicha cuadrícula estaba siendo redescubierta y revalorada en la cultura urbana y arquitectónica local e internacional. Aquel redescubrimiento señalaba un nuevo vínculo entre las intervenciones arquitectónicas y la ciudad, al pensar las primeras en relación con la estructura del tejido de las segundas. A su vez, daba cuenta de un nuevo vínculo entre la arquitectura y la historia, a partir del entendimiento de las nuevas intervenciones como parte de la ciudad existente y de su tejido, a diferencia y en clara discusión con lo que consideraban la visión universalista y ahistórica de cierta planificación urbana. Sin embargo, por motivos económicos, aquel mirador que ataba la pieza a la cuadrícula fue excluido en la construcción final.

Finalmente, en 1993, se inaugura un sector del Parque España luego de múltiples problemas vinculados con su financiamiento y con la propiedad de la tierra. ${ }^{7}$ El financiamiento sería cubierto por el gobierno de España en el marco de la celebración del Quinto Cen-
4- El uso de vegetación local coincidia y también hacía referencia a especies presentes en el territorio español. Si bien el anteproyecto referencia elementos del contexto local, otros aspectos aludian a lo español, como por ejemplo aquellas columnas que nada sostienen, remitiendo al mito de Hércules, retomado previamente en distintos escudos de armas y banderas, ya sea españolas o de algunas de sus comunidades regionales. En la misma dirección, una serie de monumentos que ervención no solo remitían a figuras españolas, sino también a idénticos monumentos existentes en España.

5- Conceptos como el de espacio público son especialmente polivalentes. Sus referencias a cuestiones politicas $y$ espaciales lo hacen particularmente flexible para los cularmente flexible para los usos más diversos, algunos
de los cuales se contraponen entre sí. Un análisis de los diversos usos y sentidos de la categoría de espacio público puede encontrarse en Novick (2003), Gorelik (2006) y Menazzi (2008).

6- En el año 1980 se había desarrollado un concurso regional de antecedentes para regional de antecedentes para
llevar adelante el anteproyecto. Los miembros del jurado eran Bohigas, Martorell y Martín Ledesma, del Centro de Arquitectos de Rosario (CAR). Fueron seleccionados el Estudio H para paisaje y vialidad y Horacio Quiroga $\&$ Asociados para el proyecto ejecutivo. 
7- Una vez concluido el anteproyecto, MBM se desentiende en gran parte de la operación, quedando la gestión a cargo quedando la gestion a cargo de actores locales que se
vincularán en distintos modos y momentos con actores españoles a los fines de avanzar en el financiamiento.

8-Serála empresa constructora española Dycasa la que lleve adelante la construcción del edificio del Parque España.

9- De las 12 héctareas del anteproyecto de $M B M$ solo se construye el sector este. se construye el sector este.
Posteriormente diversas intervenciones avanzarán en la recuperación de la costa pero sin seguir los lineamientos del anteproyecto catalán.

10- Una temprana perspectiva crítica en torno a distintos aspectos del planeamiento estratégico, las nuevas relaciones entre economía y cultura y las analogías entre el ámbito de la ciudad y el de las empresas puede encontrarse en Vainer $(2000$. contrarse en Vainer $(2000$, 2000a), Arantes (2000, 2000 ${ }^{a}$, los cuales pueden añadirse los trabajos de Lima Junior (2010). Entre los textos apologéticos de la planificación estratégica puede mencionarse a Fernández Güell (1997), y a Borja y Castells (1996), siendo Borja, como veremos, un activo consultor y difusor de la planificación estratégica y la experiencia catalana. En reiteradas ocasiones, muchas veces a partir de agencias multilaterales de crédito, Borja y otra pléyade de consultores mayoritariamente catalanes recorrerán diversas ciudades latinoamericanas asesoranlatinoamericanas asesoranla implementacion de estas perspectivas. tenario de la llegada de Cristóbal Colón a América (1492-1992). ${ }^{8}$ En efecto, aquellas celebraciones se ligaban a cambios previos en la política exterior española (Del ARENAL, 1994), que incluían como objetivos la entrada de España a la Unión Europea y las celebraciones del Quinto Centenario (1992) como parte importante de un nuevo vínculo entre España y América Latina. Luego de la finalización de la construcción de un sector más reducido de lo contemplado en el anteproyecto de $\mathrm{MBM}^{9}$, la Secretaría de Planeamiento en Rosario continuaría recuperando sectores de la costa de Rosario a partir de proyectos de arquitectura, insertos en planes más amplios, tales como el Plan Director de Rosario y el Plan Estratégico de Rosario.

\section{BUENOS AIRES Y PUERTO MADERO: INGRESO Y LEGITIMACIÓN DEL CAPI- TAL PRIVADO EN LAS GESTIONES URBANAS}

Como vimos, el Parque España y la perspectiva de intervención de BoHIGAs se vinculaban desde una mirada arquitectónica con una serie de críticas a la planificación urbana. En efecto, la estrategia del proyecto urbano, entendiendo la ciudad como la suma de sus partes, favoreciendo las intervenciones limitadas y fragmentarias ligadas al diseño urbano y los proyectos de arquitectura, impugnaba la estrategia del plan propia de la planificación urbana, la cual contaba con una visión global de la ciudad, ligada a la actuación de equipos interdisciplinarios. A su vez, como vimos, el anteproyecto de MBM en Rosario precede temporalmente el "éxito" catalán y la posterior "catarata" de difusores internacionales de aquella experiencia.

Posteriormente, en Buenos Aires, las críticas a la planificación urbana tomarán otras direcciones. Aunque también vinculadas con experiencias y debates ligados al entorno español, en este caso las críticas y los referentes no provendrán única ni principalmente del ámbito del diseño urbano. Las nuevas perspectivas críticas a la planificación urbana se relacionarán con la planificación estratégica, los acuerdos entre actores públicos y privados y las posiciones ligadas a la "ciudad de los promotores" (HALL, 1996). Entre otros actores, cabe señalar un grupo de asesores y consultores españoles que retoman debates y experiencias desarrollados en Estados Unidos en torno a una serie de equivalencias entre el ámbito de la ciudad y el de la empresa. ${ }^{10}$

En términos más amplios, a propósito de experiencias de intervenciones portuarias en Baltimore, Boston y Londres, y en torno a la idea de "ciudad de los promotores", investigadores como Peter Hall sintetizan con precisión algunos de los cambios que estas nuevas perspectivas suponen: 
Del parque España a Puerto Madero: circulación del "urbanismo de los arquitectos" y la planificación estratégica entre Argentina y España (1979-1993)

“... En los años setenta, el urbanismo cambió totalmente y en los ochenta parecía abocado a la autodestrucción. Daba la sensación de que la planificación convencional y el uso de planes y normas para reglamentar el suelo habian caído en total descrédito. En lugar de regular el crecimiento urbano, el urbanista se había dedicado a fomentarlo con todos los recursos que tenía a su alcance. La idea que predominaba era que la ciudad era una máquina de crear riqueza y que la función principal del urbanismo era engrasar la maquinaria. El urbanista se había identificado cada vez más con el promotor, su tradicional adversario: el guardabosques se había convertido en cazador furtivo...” (HALL, 1996: 354).

Perspectivas vinculadas con las descritas por Hall, asesores catalanes de por medio, irán ingresando en el medio local hacia fines de la década del 80. Un aspecto central de estas tiene que ver con su apelación a la cooperación entre actores públicos y privados en las gestiones urbanas, aunque esta posibilidad quedaba abierta una vez que experiencias centradas en lo disciplinar, como la del "Concurso de ideas urbano-arquitectónicas" (1986), propugnaban la participación de la sociedad civil y también la del mercado, aunque no encontrasen en ese entonces los capitales privados necesarios para respaldar las intervenciones urbano-arquitectónicas propuestas. ${ }^{11}$

La operación de Puerto Madero cobra un valor especial respecto de la participación del capital privado en las gestiones urbanas, aunque otras experiencias previas también iban señalando esa dirección. Los estudios en torno al puerto que desde la Facultad de Arquitectura y Urbanismo (FAU) realizaba el taller Borthagaray (s/f) introducían en el medio local una serie de conceptos ligados a la urbanística de los promotores y a las experiencias de transformación de los puertos de Baltimore, Boston y los docks de Londres. ${ }^{12}$ Pero, como veremos y a diferencia de la operación Puerto Madero, en aquellas propuestas se prioriza en parte el interés público propiciando un sistema de controles y contrapesos mediante la licitación a privados para construcción y uso por tiempo determinado, sin enajenación de los edificios ni de la tierra, que seguirían siendo públicos.

\subsection{De la regulación a la promoción: la Corporación Antiguo Puerto Madero y el Plan estratégico Antiguo Puerto Madero}

A propósito de la operación Puerto Madero cabe señalar el modo en que ingresan con mayor fuerza una serie de elementos ligados a la urbanística de los promotores y la planificación estratégica corriendo el eje aunque retomando elementos de la discusión disciplinar. Uno de los puntos clave en esa dirección es el desarrollo en 1989 de la Corporación Antiguo Puerto Madero (CAPMSA), la cual:
11-El Concurso "Ideas Urbanoarquitectónicas para Buenos Aires", más conocido como "20 Ideas para Buenos Aires", fue organizado en 1986 a partir de un convenio de cooperación entre la Municipalidad de la ciudad de Buenos Aires y la Comunidad de Madrid. Orientado especificamente a la búsqueda de ideas urbanoarquitectónicas para una serie de fragmentos urbanos de la ciudad, discutiendo explicitamente con perspectivas de intervención global, el concurso sostenía a tesis de que las soluciones a los problemas de la ciudad pasan en último términopor una reconfiguración fisica (Leira, 1988). El propio nombre de aquel concurso hace referencia a las "50 ideas para Madrid", siendo el responsable del programa por la parte española del convenio de cooperación Eduardo Leira, quien habia sido director de la Oficina Municipal del Plan Ayuntamiento de Madrid, y posteriormente continuaría vinculándose, a partir de asesorías, consultorias y convenios de cooperación, con diversas ciudades latinoamericanas. Los vínculos entre el espacio público y la movilización de capitales privados se explicitaban en la propia convocatoria, al señalarse que toda actuación sobre el espacio público genera una acción movilizadora de otros recursos públicos y privados (Municipalidad de la Ciudad de Buenos Aires, 1986). 


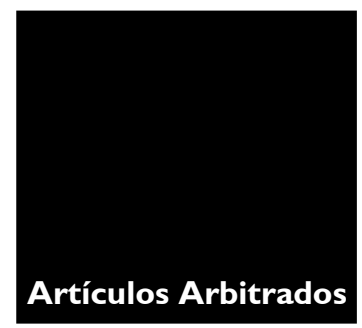

Guillermo Jajamovich

12-Los ejercicios en torno al puerto fueron desarrollados por el taller de arquitectura Borthagaray de la Facultad de Arquitectura y Urbanismo durante 1986 y 1987. Aquellos ejercicios se apoyaban en un convenio firmado en 1985 entre la Facultad de Arquitectura y Urbanismo (FAU) y la Secretaría de Transporte del Ministerio de Obras y Servicios Públicos del Gobierno Nacional.
“... tendría por objeto la confección de un plan maestro de desarrollo urbano, el estudio de la infraestructura a incorporar y la promoción de inversiones en el área, la actividad inmobiliaria y la construcción de obras nuevas y/o remodelaciones en la zona de las obras necesarias para convertirla en un polo de desarrollo urbano basado en una genuina inversión, con participación de capitales nacionales y extranjeros, como asimismo la venta y/o locación de las tierras pertenecientes al área en cuestión (...) Que, asimismo, se cumplimentarian y se realizarian efectivamente los objetivos de racionalización administrativa del Estado y de participación del sector privado nacional y extranjero en los emprendimientos productivos, que han sido fijados por el Gobierno de la Nación..." (Poder Ejecutivo Nacional, 1989).

Perspectivas de sectores del Parque España y mirador que ata la pieza a la cuadrícula. Fuente: Revista bio, marzo 1988, Rosario

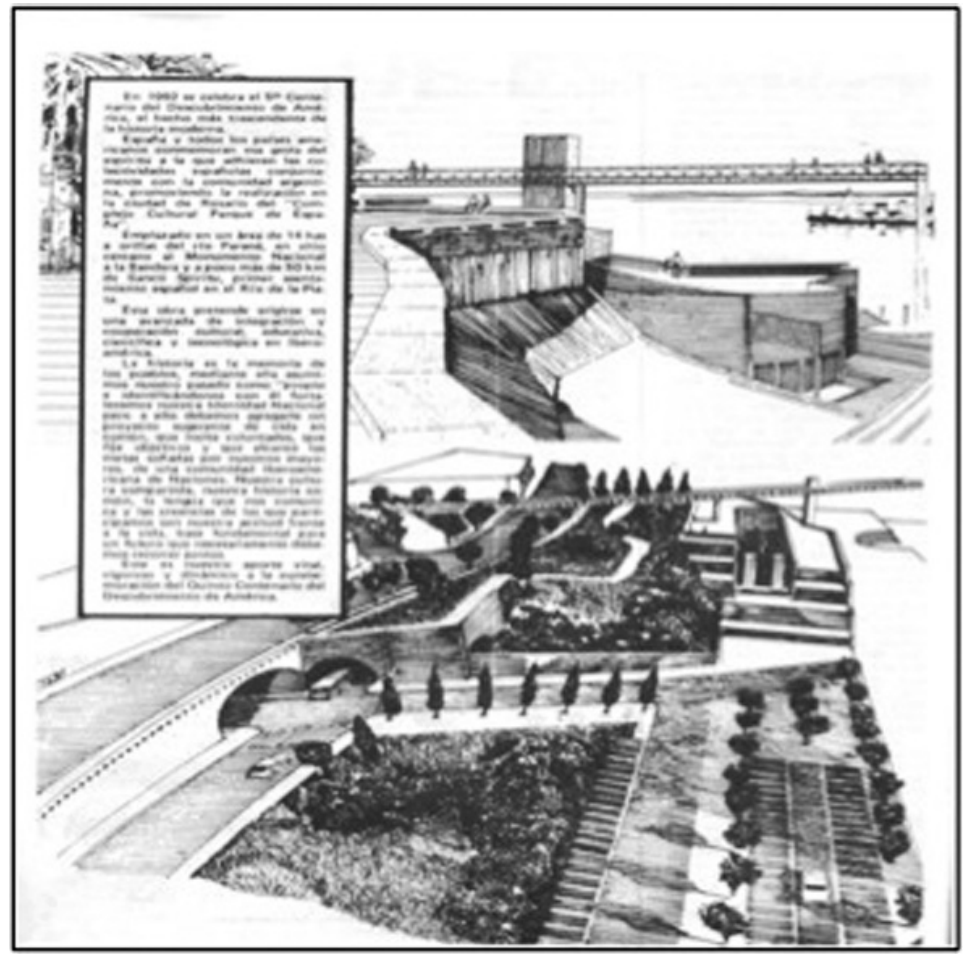


Del parque España a Puerto Madero: circulación del "urbanismo de los arquitectos" y la planificación estratégica entre Argentina y España (1979-1993)

Previamente, otras propuestas en torno al puerto no habían podido resolver los problemas jurisdiccionales. Por diversos motivos, y a lo largo del tiempo, los gobiernos locales, nacionales y los intereses privados no habían podido acordar qué hacer y cómo revitalizar el área. La CAPMSA pudo resolver aquellos problemas de gestión en tanto estaba integrada por representantes del gobierno nacional y local. Pero a su vez, presentaba explícitamente una modalidad público-privada de gestión urbana que formaba parte activa de las políticas económicas neoliberales llevadas adelante durante la presidencia de Carlos Menem. ${ }^{13}$

Otro elemento novedoso y que se vincula nuevamente con el escenario internacional tiene que ver con el encargo de un masterplan para Puerto Madero a un equipo catalán, reactivando un convenio entre Buenos Aires y Barcelona firmado en 1985. En efecto, al convenio de 1985 se le agrega un punto que incluye “... la elaboración del Plan de Puerto Madero por medio de un equipo de Consultores de Barcelona, por encargo del Ayuntamiento y del Puerto Autónomo de Barcelona y la asesoría de TUBSA..." (BorJA, 1990:

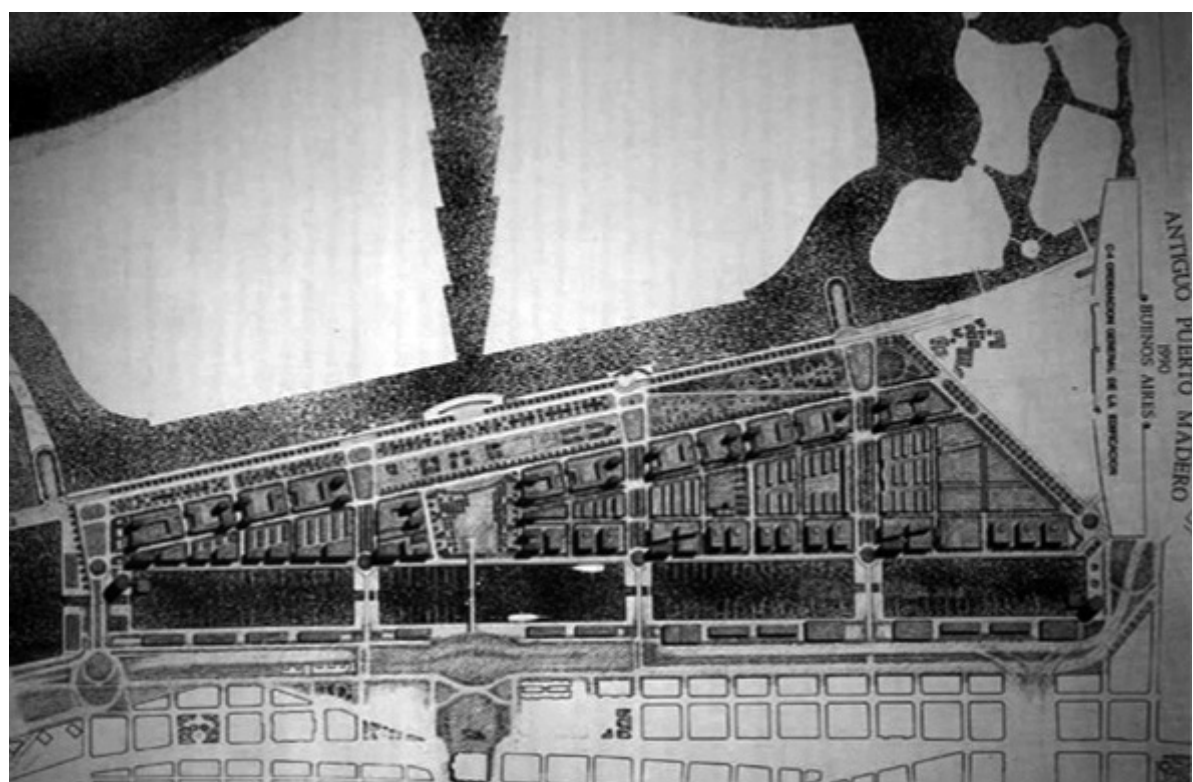

Plan Estratégico Antiguo Puerto Madero. Fuente: Consultores Europeos Asociados (1990)
13- Durante la primera presidencia de Carlos Menem (1989-1995) se desarrolló una serie de drásticas reformas económicas y politicas que incluian la reforma del Estado y la privatización de numerosas empresas estatales. Posteriormente al inicio de la operación Puerto Madero, los procesos de privatizaciones harian emerger, junto al aumento de inversiones extranjeras directas (IED), los capitales privados que habian brillado por su ausencia en la década del 80, y la demanda por parte de estos de nuevos espacios donde radicar empresas (Ciccollela, 1999). Si bien el presente articulo se centra en la circulación de ideas urbanas entre España y Argentina, cabe señalar - aunque no podamos extendernos aquí- que a partir de los mencionados procesos de privatización de empresas estatales ingresan importantes capitales españoles. Así, en diferentes porcentajes, capitales de origen español participarán en empresas privatizadas de servicios de provisión de agua y servicios cloacales, distribución de gas, energía eléctrica, telecomunicaciones $y$ sistemas de transporte y redes de acceso a la ciudad de Buenos Aires (CEDEM, 2001). En esta última privatización, puede mencionarse la participación de la misma empresa que finaliza el edificio del Parque España: la constructora española Dycasa. 
14- La empresa consultora catalana "Tecnologías urbanas de Barcelona Sociedad Anónima" (TUBSA) era presidida por Jordi Borja.

15- Cabe señalar que mientras la presencia previa de Bohigas en Rosario se vinculaba con el terreno del diseño urbano, los nuevos consultores se centrarían en el ámbito de la gestión y el management urbano.
10). ${ }^{14}$ Aquella propuesta que solicitaba una asesoría en torno al diseño urbano y la gestión para "la recuperación de Puerto Madero", que el intendente de la ciudad de Buenos Aires, Carlos Grosso, dirigió al Ayuntamiento de Barcelona, sería asumida por el Ayuntamiento y el Puerto Autónomo y derivada a la firma Consultores Europeos Asociados (CEA). Dirigida por el arquitecto Joan Busquets y el economista Joan Alemany, CEA contará en esta oportunidad con la colaboración de Jordi Borja y Jordi Domingo, y el apoyo de un equipo de Buenos Aires dirigido por Alfredo Garay y Jorge Moscato. Presentada en julio de 1990 con el título "Plan estratégico de Antiguo Puerto Madero" (PEAPM), la propuesta catalana generó resistencias en el medio local. ${ }^{15}$

El PEAPM reiteraba algunos tópicos ya mencionados en estudios y experiencias previas sobre el puerto. Así, se consideraban la cuestión del vínculo de la ciudad con el río, la costanera como espacio verde, el Puerto como ampliación del centro, la oferta de espacio residencial y de espacio terciario, la reutilización de los galpones y del patrimonio arquitectónico y la cuestión de la accesibilidad al área. Sin embargo, su novedad consistía en el fuerte ingreso de los lineamientos de la urbanística de los promotores, ligada entre otros aspectos a la intensidad de la ocupación del suelo a partir de usos vinculados con la oferta residencial de calidad alta e intermedia, y a la oferta de espacio terciario que permitiera viabilizar económicamente el proyecto, dirigiéndolo a determinados sectores sociales de alto poder adquisitivo.

Otros lineamientos propios de la urbanística de los promotores tenían que ver con la ingeniería de gestión adecuada al desarrollo de proyectos de este tipo, sintetizada en dos ejes. Por un lado, a partir del gran tamaño de la transformación, la necesidad de absorberla con operaciones de escala controlable. Por otro lado, la cuestión de abordar el tema a partir de la iniciativa pública con inversión privada. Otra diferencia fundamental respecto de proyectos anteriores es el sustento político-institucional que respalda esta propuesta. El antecedente de la creación de la CAPMSA y el encargo del masterplan catalán surgían de la iniciativa del máximo representante político de la ciudad de Buenos Aires, el intendente Carlos Grosso, en acuerdo con el entonces presidente Carlos Menem.

Las formas de organización y gestión propuestas por el PEAPM retoman los lineamientos establecidos por la CAPMSA. Como vimos, estos señalaban la posibilidad de venta, alquiler o concesión del suelo o de las construcciones nuevas o existentes. Así, el PEAPM señalaba la predominancia de los mecanismos de concesión y venta de suelo urbanizado por parte de los organismos de gestión de aquellos emprendimientos en otras experiencias internacionales. El PEAPM sostenía que construir o rehabilitar edificios por parte de la 
Del parque España a Puerto Madero: circulación del "urbanismo de los arquitectos" y la planificación estratégica entre Argentina y España (1979-1993)

organización responsable de la reconversión portuaria no era en ninguno de los casos una de sus responsabilidades. Así, excluía la alternativa de alquiler y la posibilidad de intervenir directamente en la construcción o rehabilitación de edificios. Entonces, según el PEAPM, la CAPMSA debería escoger entre dos formas básicas de gestión del suelo: concesión o venta. En el caso de Buenos Aires, el PEAPM sugiere que la consideración exclusiva del sistema de venta del suelo y de las edificaciones de Puerto Madero sería un gran error. Como alternativa de gestión adecuada, sostienen la necesidad de una combinación de los sistemas de venta y concesión del suelo.

\subsection{Reacciones al proyecto catalán: rechazo a la propuesta urbana y mantenimiento del} sistema de gestión

Presentada públicamente en julio de 1990, la propuesta catalana generó resistencias en el medio local, a partir de las cuales se retomaron una serie de discusiones en torno a los vínculos entre plan y proyecto, actores públicos y privados y actores locales y extranjeros, entre otras temáticas. A pesar de los apoyos políticos-institucionales a partir de los cuales se desarrolla el PEAPM, se produce una serie de reacciones a este. El epicentro de la confrontación con el PEAPM se desarrolla en torno a la Sociedad Central de Arquitectos (SCA), la cual manifiesta su rechazo a partir de numerosas mesas-debate, artículos y solicitadas publicadas en revistas disciplinares y en periódicos de gran difusión. Entre otros aspectos, la sociedad de arquitectos critica la falta de participación de arquitectos locales y de un concurso para aquella operación, junto a la inexistencia de un plan global que la incluyera, denunciando su carácter inmobiliario.

La SCA sintetiza sus posturas críticas en cuatro aspectos: el programa, el proyecto urbano, la ecuación económica y la gestión (KeSELMAN y DEL Franco, 1991). Así, postulan que el programa urbanístico adoptado no se ha fundamentado, ignorándose los problemas del puerto en su déficit de superficie, la falta de superficies recreativas y de análisis del impacto del proyecto en las áreas urbanas próximas. Respecto del proyecto urbano, se postula su incongruencia al proponer un distrito de intensas actividades centrales, que incluye en su interior una franja de viviendas de características suburbanas. A su vez, se critica que no se plantee el problema de las barreras que suponen el ferrocarril y la autopista a la hora de lograr una integración urbana del área respecto de la ciudad. Se postula, asimismo, la falta de un plan armónico y factible de desarrollo del proyecto en etapas. En relación con la ecuación económica, se plantea la ambigüedad de los postulados, ya que si por un lado se expresa que la CAPMSA debe asumir la construcción de la infraestructura antes de poner los terrenos a la venta, por otro lado, se incluye su posibilidad de venta en los primeros 
16- Los asesores del concurso eran integrantes de la MCBA $y$ de la SCA. Participaron 93 estudios de arquitectos de la Argentina y fueron premiados tres proyectos. De estos tres equipos saldría conformado uno nuevo, más reducido, encargado de producir el proyecto definitivo. Entre los diversos puntos señalados por el jurado, interesa remarcar el modo en que se hace énfasis en la cuestión de la gestión de los proyectos. Así, se postula que se tuvo en cuenta la búsqueda de relación entre las propuestas y las tendencias reales de inversión urbana en aquella coyuntura. De ese modo, junto a la valorización espacial de los proyectos, se evaluaba la cuestión de las diversas escalas de inversión y los modelos alternativos de gestión propuestos. La elección del equipo redactor se produce en febrero de 1992 $y$ el proyecto definitivo, en octubre de 1992. Previamente a los resultados del concurso, en septiembre de 1991, se había comenzado la licitación de una serie de galpones de Puerto Madero. años de la operación. En tanto crítica a la gestión propuesta, se indica que por un lado se habla de venta de terrenos, y por otro, se señala la conveniencia de una modalidad de concesión del suelo. Sintetizando su postura, la SCA postula que el proyecto no ha alcanzado el grado de madurez que permita iniciar acciones y que su factibilidad económica no está garantizada. Así, recomienda pausar el progreso de las operaciones, reconsiderando el programa, el proyecto y las estrategias de implementación, en un marco de participación y estudios más amplios y consensuados.

Ahora bien, ¿qué significa en esa coyuntura un marco de participación y estudios más amplios y consensuados? Junto a los arquitectos proyectistas, que tenían críticas puntuales al proyecto en tanto tal, y que, sobre todo, querían participar de la iniciativa a través del concurso urbanístico y a través de la construcción de edificios (GORELIK, 2003), GorELIK sintetiza otras dos líneas de debate, alrededor de distintos núcleos argumentales. En primer lugar, el de la planificación urbana (SUÁREZ, 1990), para el cual las funciones portuarias debían ser garantizadas, las funciones para todo el sector debían ser las administrativas centrales y las recreativas y las residenciales solo debían ser complementarias (GoRELIK, 2003). El segundo sector era aquel representado por el secretario de planeamiento Alfredo Garay, quien presentó el masterplan como una formulación de escenarios alternativos para una ingeniería de la gestión (GORELIK, 2003).

La extensa serie de críticas formuladas por la SCA será finalmente "neutralizada" por parte de la gestión Grosso-Garay. Esta logrará la participación de los arquitectos locales a partir de la suspensión del masterplan catalán y la organización y el llamado a un concurso de ideas organizado por la propia SCA, en julio de 1991 y en acuerdo con la Municipalidad de la Ciudad de Buenos Aires (MCBA). Así, la postulación de la necesidad de un plan que le dé sentido a la operación, así como las críticas que apuntaban al carácter inmobiliario y excluyente de esta, dejan de ser sostenidas por la representación de los arquitectos, aunque cabe señalar que la intensidad del uso de la tierra disminuirá respecto de la propuesta catalana (de cerca de 3.000 .000 a $1.500 .000 \mathrm{~m}^{2}$ edificables). ${ }^{16}$ La gestión urbana a cargo de Grosso-Garay logra insertar a la SCA dentro del proyecto, abandonando el masterplan en tanto proyecto urbano pero manteniendo y fortaleciendo la perspectiva ligada a la participación del capital privado en la ciudad, relegitimada esta vez a partir de la participación de arquitectos locales, y conservando el aporte principal de la consultoría española: un sistema de gestión con iniciativa y control público y con actuación y financiación privada. 
Del parque España a Puerto Madero: circulación del "urbanismo de los arquitectos" y la planificación estratégica entre Argentina y España (1979-1993)

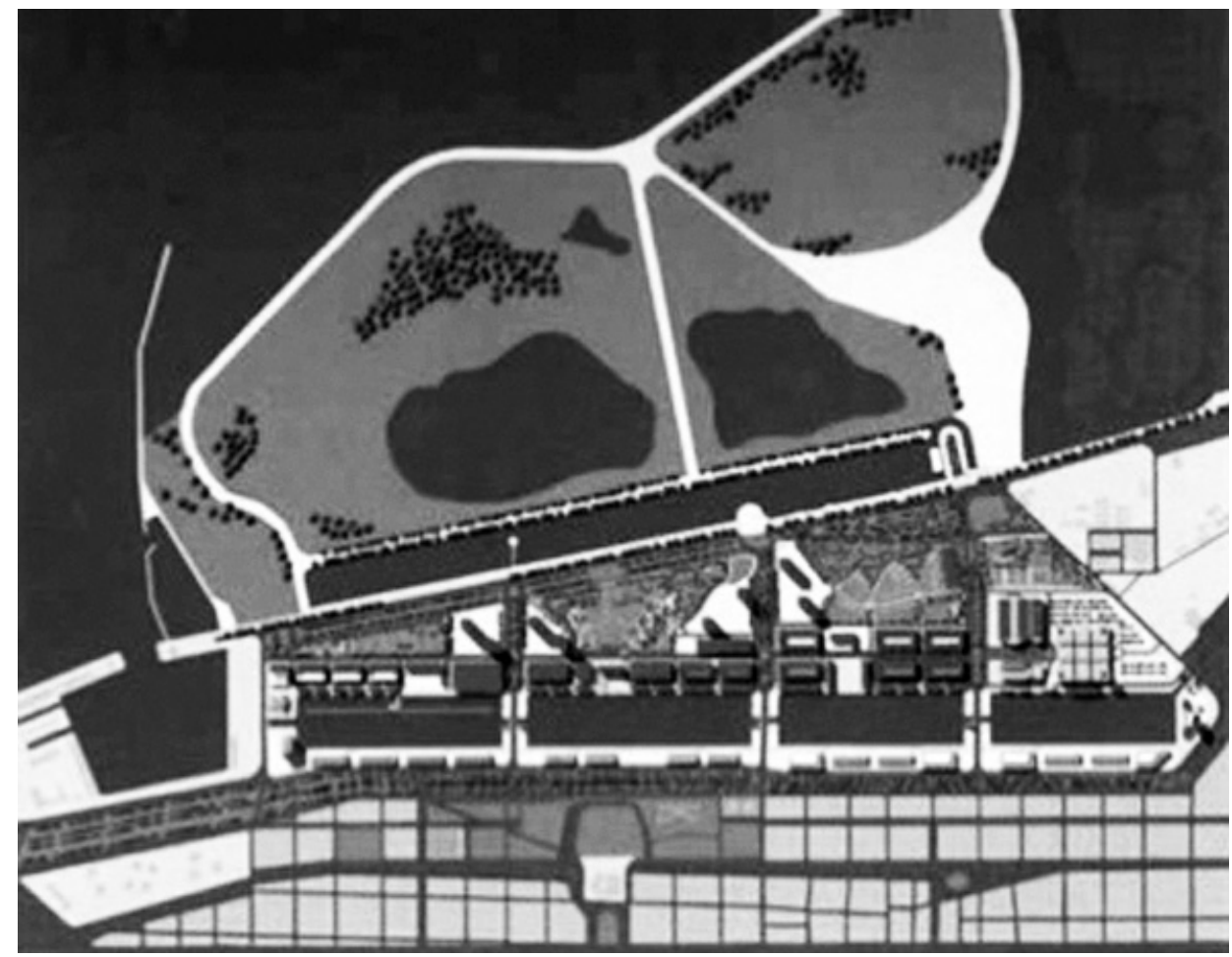

Proyecto definitivo para Puerto Madero. Fuente: http://www. puertomadero.com historiab.cfm

4. CONCLUSIONES: ROSARIO, BUENOS AIRES Y LA CIRCULACIÓN DE LA PLANIFICACIÓN ESTRATÉGICA

Investigadores como GoRELIK $(2003,2004)$ señalan los diversos modos en que las perspectivas ligadas a la planificación estratégica han funcionado en Barcelona, en relación con el modo en que efectivamente se desarrollaron en ciudades como Buenos Aires. Frente a perspectivas como la de ArANTES (2000, 2000a, 2000b), que cuestiona en bloque el desarrollo y la aplicación de la planificación estratégica, tanto en sus contextos de origen como en sus contextos de recepción, GoRelik (2004) señala que mientras en su contexto de origen, con la presencia activa de políticas estatales y una vivificada cultura urbana, 
17- Según Isabel Martinez de San Vicente (1998), la operación Parque España es la primera avanzada en la caracterización arquitectónica de la costa de Rosario. pudieron cumplir un rol 'progresista', en su contexto de recepción, como en el caso de Buenos Aires, funcionaron como aliadas de la fragmentación urbana.

No es esta la ocasión para discutir la posición de GoRELIK acerca del supuesto carácter progresista de la planificación estratégica en su contexto de producción. Pero sí interesa retomar su perspectiva que señala la posibilidad de que en su circulación internacional ideas tales como aquellas vinculadas con la planificación estratégica puedan ir tomando sentidos diversos al insertarse en contextos con tradiciones y configuraciones de actores distintas.

En ese sentido, interesa señalar que el ingreso de la planificación estratégica en Rosario tuvo un funcionamiento diferente del apuntado en Buenos Aires, insertándose en un contexto en el cual las tradiciones públicas y las políticas urbanas cuentan con una mayor cohesión y continuidad en el interior de los gobiernos municipales, al menos en el período aquí analizado. Sin embargo, previo al desarrollo del Plan Estratégico de Rosario (PER), cuyo inicio data de 1998 habiendo participado de él asesores catalanes como Jordi Borja y Joan Alemany, puede señalarse la experiencia del Parque España como un ejemplo de planificación estratégica "avant la lettre", es decir, previo a la difusión explícita de aquellas perspectivas en las que otro grupo de consultores catalanes jugó un rol relevante.

Se trata, creemos, de una anticipación que incluye asimismo cambios sustanciales en relación con lo que luego sería la retórica de la planificación estratégica. Por un lado, el proyecto del Parque España funcionó en parte como la posterior planificación estratégica postula que deberían hacerlo los proyectos urbanos: como puntapié inicial a una serie de iniciativas publico-privadas que permitan transformar el territorio ${ }^{17}$, aunque en este caso, a diferencia de Buenos Aires, el predominio está del lado de lo público y no de lo privado, ligado a usos recreativos, culturales y educativos, si bien un sector del edificio del Parque España fue cedido funcionando allí un colegio español privado.

Pero cabe remarcar una peculiaridad de aquel proyecto en lo que a vínculos entre sectores públicos y privados se refiere. Se trata de un aspecto que las miradas celebratorias de la experiencia del Parque España y de la ciudad de Rosario tienden a dejar de lado. Si en la retórica de la planificación estratégica la alianza de actores públicos y privados funciona como una necesidad a la hora de alcanzar consensos mayores y efectivizar los proyectos, en el caso del Parque España los actores que apoyan la iniciativa no parecen responder del todo a aquellos ejes. En efecto, la que inicialmente moviliza el proyecto será una asociación 
Del parque España a Puerto Madero: circulación del "urbanismo de los arquitectos" y la planificación estratégica entre Argentina y España (1979-1993)

no estatal sin fines de lucro como la Federación de Entidades Españolas de la provincia de Santa Fe, encontrando un eco favorable, y aquí aparece una diferencia relevante respecto de los futuros planteos de la planificación estratégica, en una gestión municipal de la época de la dictadura militar, la cual decide entregar las tierras correspondientes para el desarrollo del proyecto. ${ }^{18}$ Sin embargo, como vimos, la factibilidad financiera-económica del proyecto se producirá recién en democracia, cuando el gobierno de España decida financiarlo en cooperación con el gobierno ya democrático de la ciudad de Rosario, en dirección a los festejos del quinto centenario.

A lo largo del presente trabajo he ido señalando cómo los vínculos entre política e intervenciones urbanas no pueden establecerse de antemano. Diferentes entramados de actores y diversos contextos culturales, económicos y políticos hacen que ideas similares del debate internacional tengan efectos sociales y espaciales distintos en ciudades como Buenos Aires y Rosario. Esta característica se complejiza aun más cuando se consideran los procesos de circulación internacional de ideas. Por ejemplo, como vimos, en España las perspectivas críticas a la planificación urbana desarrolladas desde un punto de vista arquitectónico se vinculaban con los nuevos ayuntamientos democráticos. Sin embargo, en Rosario, el anteproyecto de MBM introduce estas posiciones en un contexto de restricciones democráticas. En la misma dirección, propuestas, proyectos y consultorías ligadas al entorno catalán tendrán efectos distintos en Puerto Madero y Parque España.

\section{BIBLIOGRAFÍA}

ARANTES, Otilia (2000) "Uma estratégia fatal. A cultura nas novas gestões urbanas". En: ARANTES, Otilia; VAINER, Carlos y MARICATO, Erminia. A cidade do pensamento único. Desmachando consensos. Vozes, Petrópolis.

ARANTES, Otilia (2000a) "Cultura y coaliciones de poder y dinero en las nuevas gestiones urbanas". Block, número 5, pp. 12-21, Buenos Aires.

ARANTES, Otilia (2000b) "Pasen y vean... Imagen y city marketing en las nuevas estrategias urbanas". Punto de Vista, N. ${ }^{\circ}$ 66, pp. 16-19, Buenos Aires.

BORJA, Jordi (1990). "Presentación”. En: Consultores Europeos Asociados. Plan Estratégico de Puerto Madero. Buenos Aires.

BORJA, Jordi \& CASTELLS, Manuel (1996) Local y global: la gestión de las ciudades en la era de la información. Taurus, Madrid.

BOURDIEU, Pierre (2000) "Las condiciones sociales de la circulación de las ideas". En: Intelectuales, política y poder. Eudeba, Buenos Aires.
18- Excede el objeto del presente trabajo pero no puede dejar de mencionarse el proyecto de $M B M$ en el año 1981 para la remodelación de ciertas áreas del Parque Sarmiento en la ciudad de Córdoba. Aunque no supera la etapa proyectual, cabe señalar que fue nuevamente solicitado a MBM en un contexto de dictadura militar. En este caso, la demanda del proyecto a MBM se relaciona con los vínculos de Bohigas con el entonces secretario de obras públicas de la Municipalidad de Córdoba, el arquitecto Miguel Ángel Roca. 
CEDEM (2001). "La propiedad del capital de las empresas privatizadas de servicios públicos que actúan en la Ciudad de Buenos Aires: principales características y transformaciones recientes". En: Coyuntura económica de la ciudad de Buenos Aires. N. ${ }^{\circ} 2$, Buenos Aires.

CICOLELLA, Pablo (1999). “Globalización y dualización en la Región Metropolitana de Buenos Aires. Grandes inversiones y reestructuración socioterritorial en los años noventa". EURE, v. 25, n. 76, p. 5-27, Santiago de Chile.

CONSULTORES EUROPEOS ASOCIADOS (1990) Plan Estratégico de Antiguo Puerto Madero. Buenos Aires.

DEL ARENAL, Celestino (1994) La política exterior de España hacia Iberoamérica. Editorial Complutense, Madrid.

ELGUEZABAL, Eduardo (1979). “Oriol Bohigas en Rosario”. Summa, N. 140, Buenos Aires.

ESTUDIO MBM, MARTOREL, BOHIGAS, MAC KAY; ESTUDIO H; QUIROGA, Horacio y asociados, arqs. (1983). "Parque España, Rosario". Summa, Colección Temática, 3/83, Buenos Aires.

FERNÁNDEZ GUELL, José Miguel (1997) Planificación Estratégica de Ciudades. Gustavo Gili, Barcelona.

GORELIK, Adrián (2006). “El romance del espacio público”. Block, 7, 8-15.

GORELIK, Adrián (2004). "Para una agenda política de reformas urbanas". En: Miradas sobre Buenos Aires. Historia cultural y crítica urbana. Siglo XXI editores, Buenos Aires.

GORELIK, Adrián (2003). "Las ideas urbanísticas en la década de 1980 en Buenos Aires. Una historia del concurso para Puerto Madero". En: Seminarios en economía y políticas urbanas, Universidad Torcuato Di Tella, 28 de octubre, ciudad de Buenos Aires.

HALL, Peter (1996). "La ciudad de los promotores. La práctica urbanística cuestionada: Baltimore, Hong Kong, Londres, 1975-1987’. En: Ciudades del Mañana, Ediciones del Serbal, Barcelona.

KESELMAN, Julio y DEL FRANCO, Carlos (1991) y del Franco, C. (1991) Puerto Madero - Italpark: posición de la SCA. Revista de Arquitectura, 150.

LEIRA, Eduardo (1986). “¿Para qué ‘ideas’ sobre Buenos Aires?”. En: Municipalidad de la ciudad de Buenos Aires (1986) Convocatoria para el llamado a Concurso de Ideas Urbano-Arquitectónicas para Buenos Aires. MCBA, Buenos Aires.

LIMA JUNIOR, Pedro (2010) Uma estratégia chamada 'Planejamento estratégico'. Deslocamentos espaciais e a atribuição de sentidos na teoría do planejamento urbano. 7 Letras: Río de Janeiro.

MARICATO, Herminia (2000). “As ideáis fora do lugar e o lugar fora das ideáis". En: ARANTES, Otilia; VAINER, Carlos y MARICATO, Erminia. A cidade do pensamento único. Desmachando consensos. Vozes, Petrópolis. 
Del parque España a Puerto Madero: circulación del "urbanismo de los arquitectos" y la planificación estratégica entre Argentina y España (1979-1993)

MARTÍNEZ DE SAN VICENTE, Isabel (1998). "La sutil belleza de la ciudad aluvional. Crónica de 15 años de arquitectura pública en Rosario". Summa+ 33, pp. 110-119, Buenos Aires.

MENAZZI, Luján (2008). "Espacio público y política: un abordaje desde los discursos mediático, político y académico". Revista Question N. ${ }^{\circ} 17$. Universidad Nacional de La Plata, La Plata.

MUNICIPALIDAD DE LA CIUDAD DE BUENOS AIRES (1986) Convocatoria para el llamado a Concurso de Ideas Urbano-Arquitectónicas para Buenos Aires. MCBA, Buenos Aires.

NOVICK, Alicia (2003). "Espacios y proyectos. Oposiciones, hegemonías e interrogantes". En: Las dimensiones del espacio público. Problemas y proyectos. SP-GCBA, Akian, Buenos Aires.

PODER EJECUTIVO NACIONAL (1989) Creación de la Corporación Antiguo Puerto Madero. Decreto 1279/1989.

SAINZ GUTIÉRREZ, Victoriano (2006) El proyecto urbano en España. Génesis y desarrollo de un urbanismo de los arquitectos. Universidad de Sevilla, Consejería de Obras públicas y transportes: Sevilla.

SAINZ GUTIERREZ, Victoriano (1999) La Cultura Urbana de la Posmodernidad: Aldo Rossi y su Contexto. Alfar, Sevilla.

SUÁREZ, Odilia (1990). "Observaciones respecto al proyecto de desarrollo urbano del área Puerto Madero según fue expuesto en la mesa redonda realizada en la Sca el 7-8-90". Revista de Arquitectura, nro. 149, Buenos Aires.

TALLER DE ARQUITECTURA JUAN MANUEL BORTHAGARAY (s/f) Propuesta para la recuperación y desarrollo del área Puerto Madero-Costanera Sur y relleno sobre el río, EPFL / FAU-UBA.

VAINER, Carlos (2000) "Pátria, empresa e mercadoria. Notas sobre a estratégia discursiva do Planejamento Estratégico Urbano". En: ARANTES, Otilia; VAINER, Carlos y MARICATO, Erminia. A cidade do pensamento único. Desmachando consensos. Vozes, Petrópolis.

VAINER, Carlos $\left(2000^{\mathrm{a}}\right)$. "Os Liberais também fazem planejamento urbano? Glosas ao 'Plano Estratégico da cidade do Rio de Janeiro'”. En: ARANTES, Otilia; VAINER, Carlos y MARICATO, Erminia. Arantes, O., Vainer, C. y Maricato, E. A cidade do pensamento único. Desmachando consensos. Vozes, Petrópolis. 
